\title{
Cultura de paz y religiones abrahámicas.
}

Recibido: 3 abril 2014 / Aceptado: 29 de abril 2014

Por Anastasio Lovo ${ }^{1}$

\section{RESUMEN}

Anastasio Lovo analiza la relación entre las religiones abrahámicas, el judaísmo, el cristianismo y el islamismo y la cultura de paz. El autor destaca la praxis epistémica e histórica de la alianza entre Dios y los seres humanos para preservar la creación en paz. Deriva el análisis hacia la hipótesis de una virtual pertinencia de la fe cristiana en sus vertientes evangélica y católica, como fundamento toral de la cultura de paz para el mundo occidental.

Palabras clave: Religiones, judaísmo, islamismo, cristianismo, cultura de paz.

\section{ABSTRACT}

Anastasio Lovo analyzes the relationship between Abrahamic, Jewish, Christian and Islamic religions and the Culture of Peace. The author emphasizes the epistemic and historical praxis of God's covenant with human beings to preserve peace in all His creation. The author's analysis is derived from the hypothesis of a virtual relevance of the Christian faith in its Protestant and Catholic aspects, as a basis of the Culture of Peace for the Western world.

Key words: Religions, Judaism, Islam, Christianity, Culture of Peace

\section{Judaísmo, Cristianismo, Islam y cultura de paz}

Según Hans Kung en su obra capital, El cristianismo y las grandes religiones, una teóloga musulmana de origen pakistaní ha acertado al aseverar que: "Cada una de las tres grandes religiones del Cercano Oriente, así suele decir la teóloga islámica, Riffat Hassan, tiene un punto neurálgico concreto, que para sí misma no es negociable, pero para las otras dos es inaceptable. Tal punto es, para el judaísmo, la singular elección de Israel como pueblo de Dios (con la promesa de

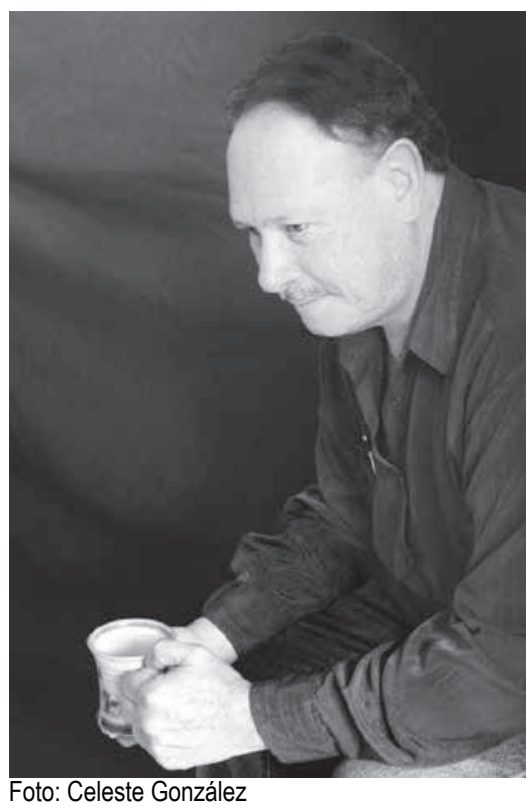
la tierra), para el cristianismo, la doctrina de Cristo como hijo de Dios, y para el Islam, la doctrina del Corán como palabra de Dios."

Estos dogmas de fe indiscutible, no negociables e insalvables para cada una de las tres religiones monoteístas, han generado conflictos sociales y guerras terribles, algunas de las cuales no han cesado hasta nuestros días. Las tres religiones monoteístas en sus textos sagrados inscriben guerra/ paz como parte del sistema cultural humano donde ocurre la revelación. La revelación misma contempla la utopía de la paz, la acción violenta para preservar o expandir la fe, sus símbolos y sus lugares sacros.

Una violencia ejercida contra la naturaleza (sacrificios animales, destrucción y contaminación ecológica) en el caso de la Alianza de Yavé con el pueblo de Israel; una violencia ejercida en contra de los humanos (las guerras de religión y las guerras geopolíticas). Así por ejemplo, Jerusalem es ciudad santa para estas tres religiones y desde hace siglos esta sacra ciudad no conoce la paz.

Para la tradición hebreo cristiana la constitución de este sistema de guerra y paz como parte de la realidad y la condición humana, está explícitamente inscrito en las palabras de Salomón -el Predicador (Cohelet)- en el capítulo 3 del libro Eclesiastés de la Biblia: El momento

1 Escritor nicaragüense, Presidente del Centro Nicaragüense de Escritores (CNE), Coordinador del Área de Cultura de Paz del Instituto "Martin Luther King", UPOLI. 


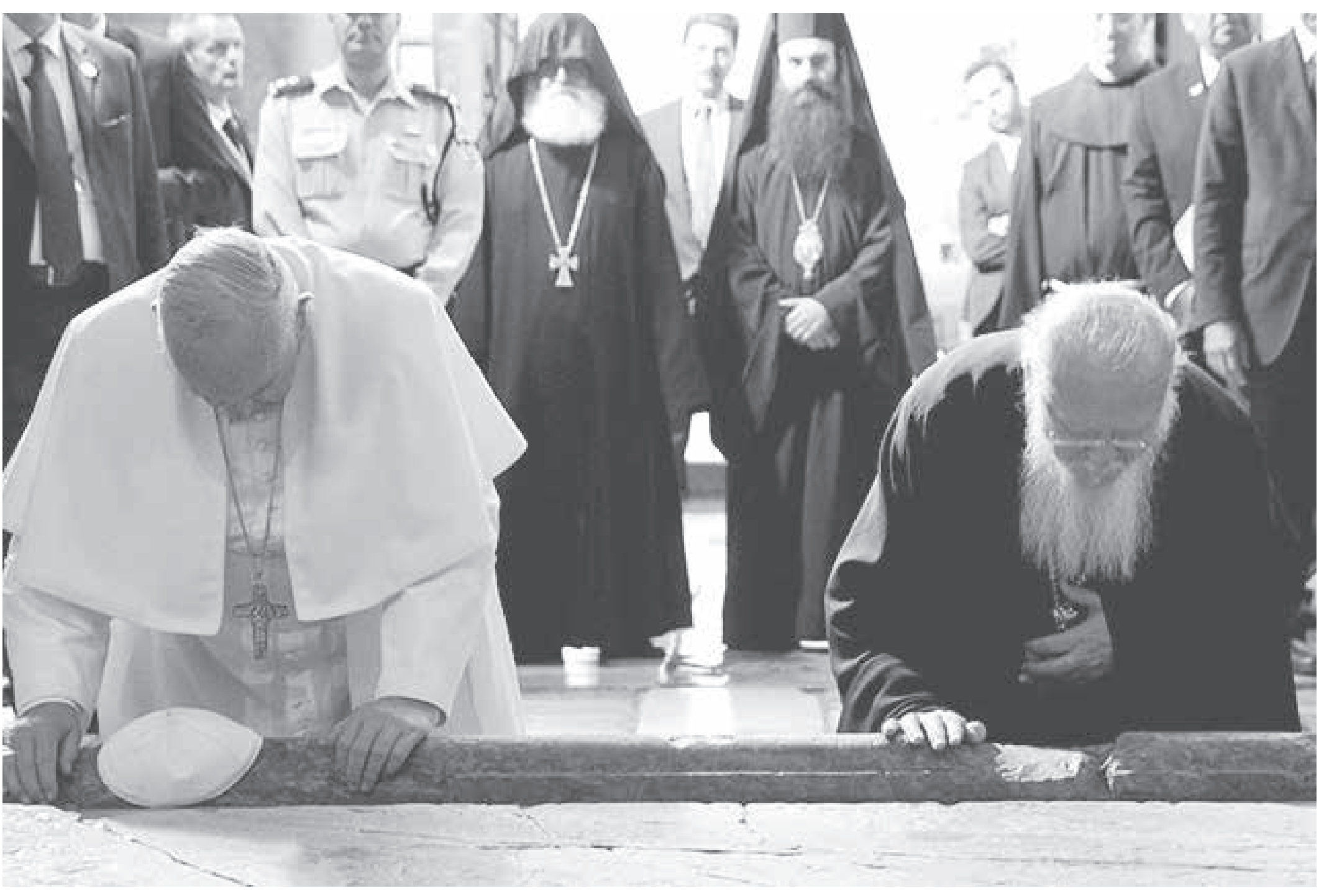

Fuente EFE

oportuno 3:1 Hay un momento para todo y un tiempo para cada cosa bajo el sol: 3:2 un tiempo para nacer y un tiempo para morir, un tiempo para plantar y un tiempo para arrancar lo plantado; 3:3 un tiempo para matar y un tiempo para curar, un tiempo para demoler y un tiempo para edificar; 3:4 un tiempo para llorar y un tiempo para reír, un tiempo para lamentarse y un tiempo para bailar; 3:5 un tiempo para arrojar piedras y un tiempo para recogerlas, un tiempo para abrazarse y un tiempo para separarse; 3:6 un tiempo para buscar y un tiempo para perder, un tiempo para guardar y un tiempo para tirar; 3:7 un tiempo para rasgar y un tiempo para coser, un tiempo para callar y un tiempo para hablar; $3: 8$ un tiempo para amar y un tiempo para odiar un tiempo de guerra y un tiempo de paz.
El Eclesiastés según los especialistas en el tema, pudo haber sido escrito en el siglo IV antes de Cristo, que corresponde al período de nacimiento de la filosofía clásica griega. Es impresionante el parentesco de contenido y formal con el fragmento N0.67 de la obra de Heráclito de Éfeso (N.535-M.475 A.C.):

"Dios es día y noche, invierno y verano, guerra y paz, hartazgo y el hambre, pero él toma varias formas, tal como el fuego, cuando se mezcla con especias, se denominan de acuerdo con el sabor de cada uno."

Pero si bien en los textos sagrados hebreos-grecocristianos-islámicos, se testimonia de manera subyacente esta episteme sistémica de guerra/paz y a prácticas violentas -sacrificios, guerras y contaminación- también 
se colocan los elementos para una superación de esta conflictividad, violencia y belicosidad. Un elemento toral en estas culturas es el de la alianza entre la divinidad y los seres humanos. Son destacables las sucesivas alianzas que Yavé ha establecido con los profetas, o la encarnación de su hijo Jesús para fundar una nueva alianza, la alianza del amor, o en el caso del Islam, el dictado de la palabra divina que Alá le hizo a Mahoma.

Dada la conflictividad humana insoslayable, la alianza funciona como un elemento destinado a disponer el ritual del sacrificio animal, la norma o decálogo a cumplir estrictamente, la encarnación sacrificial del hijo de Dios y el dictado de Alá a Mahoma de su mandato. Con el cumplimiento de este mando, se preserva la alianza.

Como afirma María José Cano referente al judaísmo, en su ensayo: El pueblo de la alianza publicado en el libro Historia de la Paz de la Universidad de Granada, España: "La sociedad hebrea articuló toda una serie de mecanismos para la regulación pacífica de conflictos en la que todos y cada uno de los componentes ejercen un papel determinado, tal como podemos comprobar a través de los textos, pero quizás el mecanismo más importante y significativo es el de las alianzas."

El creador en las tres grandes religiones monoteístas se concibe en alianza eterna -susceptible de renovarse como en el caso del cristianismo- con sus criaturas. No se concibe un demiurgo enajenado de su creación ni viceversa. Pero este eje de las alianzas entre Dios y el ser humano, dado el libre albedrío de la criatura, genera una conflictividad, agresividad, violencia y hasta guerras causadas por los apetitos de posesión sobre poder, riqueza y placer.

Estos apetitos humanos obviamente producen una masa crítica de impotentes, desposeídos e insatisfechos que se convierten en los más importantes caldos de cultivo de la violencia y la guerra. Estas dínamos de la violencia han sido ideológicamente invertidas, enmascarados y deificados por estas religiones como guerras santas (judaísmo), cruzadas (cristianismo) y jihad (guerra santa del Islam).

En el caso de la cultura helénica -matriz cultural de Occidente- los grandes reguladores de la conflictividad fueron la catarsis lograda por el arte, la racionalidad de la filosofía y la ciencia; y en política la búsqueda del bien común y la construcción de la democracia. La episteme helénica se va a integrar a la cultura occidental-cristiana. La llamada cultura occidental cristiana es producto de la síntesis lograda en la integración de elementos epistémicos- matriciales provenientes pues de las culturas hebreas, greco-latina y cristiana. Para el caso de los países de la península ibérica habría que añadir la episteme arábica. Y en el caso de los iberoamericanos, la gran raíz cultural de los pueblos originarios de Abya Yala (América).

\section{Cristianismo y cultura de paz}

El Reverendo José Miguel Torres Pérez (QEPD) -teólogo nicaragüense- en su libro "Bases y fundamentos bíblicoteológicos de una Cultura de Paz" (Torres 2003, p.7), afirma en un acápite que subtitula como La paradoja de las bases bíblicas, lo siguiente: "En La Biblia, que es un conjunto de libros escritos en una diversidad de contextos, situaciones y circunstancias a lo largo de un período de más de 1,500 años; muchas veces encontramos bases para poder fundamentar los temas más contradictorios, o sea que habrían bases para afirmar una determinada perspectiva y también su contraria. A tal extremo, que algunas veces podemos afirmar que "es verdad lo uno, pero también es verdad lo otro"; por ejemplo, podemos decir que hay bases para afirmar la opción por la violencia y por la no-violencia; también en la dimensión de lo eskatológico, podemos decir que "el Reino viene y también que ya está aquí"; en el lenguaje teológico esto se conoce no tanto como una contradicción lógica, sino como una paradoja."

La escritura bíblica misma, matriz textual de la fe cristiana, es una clara evidencia del registro más o menos fiel de una realidad humana compleja que únicamente se puede plasmar en un cúmulo de paradojas o aparentes contradicciones. La Biblia es la palabra y la acción de Dios actuando en la historia de sus criaturas: el género humano. Este sentido de la historia existe únicamente para nosotros, la única especie capaz de relatarla oralmente o por escrito. Porque la historia solamente es producida por la única especie capaz de relatar: la humana.

El Dios de la Alianza inspira un libro -La Biblia- donde opera el Espíritu Santo sobre una materia humana muy lábil, vaga, paradójica y contradictoria a la que el mismo dotó de inteligencia y libre albedrío. Este sujeto humano 
es quien realiza -inspirado por Dios- la propia historia de la alianza de Dios con su especie. Es la comunicación de Dios con sus criaturas a través de los profetas y la comunicación-comunión de Dios y sus criaturas a través de Jesucristo, su hijo.

En lo anterior no estoy afirmando que la palabra divina sea ambigua, contradictoria o paradójica, sino que en tanto inspirada por Dios pero realizada por manos humanas es decir, escrita por mentes que gozan del libre albedrío, La Biblia resulta ser lo que es: un texto paradójico que aborda la totalidad contradictoria del ser humano. Aún en este supremo texto, Dios en su inspiración nos ha dejado en libertad. A su magnífica obra de amor -y por causa del amor- Dios la deja en libertad. No existe posibilidad de realización del amor si éste conlleva una carga de opresión. Esa es una lección fundamental en la creación divina: la libertad humana.

Las decisiones humanas tomadas en libertad, también han sido las responsables de generar o producir el sistema de la guerra/paz que también puede graficar o relatar la historia del ser humano. Los motivos que fundamentan la toma de decisiones evidentemente tienen que ver con lo vacío del ser humano en ausencia de Dios, es decir sin conocer el Amor. Vacío que el ser humano (sapiens/demens) pretende vana y vanidosamente llenar con riquezas, poder, placeres, opresiones, supresiones, adicciones, etc. Eventos humanos que indudablemente producirán siempre conflictividad, violencia y belicosidad (guerras) al interior del ser humano, de la familia, de la comunidad, de la sociedad, de las naciones y entre los estados; así como entre el ser humano por un lado; y por otro lado, las especies y los recursos naturales.

Pero el cristianismo para la cultura occidental es un componente matricial de nuestro código comunicacional. El cristianismo aporta a nuestro código de comunicación, en la esfera de los valores ideológicos, una visión de mundo ético-moral, epistémico, axiológico y estética, fundamentada en el amor (como verbo-acción) y cuyo objetivo estratégico o telelógico será sustantivarse en una praxis histórica de paz.

Sin chovinismo egocentrismo o cristiano-centrismo: el amor, la paz, la reconciliación, el perdón, la restauración, la igualdad, la libertad, la justicia y el abandono del mal social (pecado), son valores constitutivos de nuestra fe.
Valores supremos cuya concepción y praxis humanizan y divinizan a nuestra especie.

El célebre teólogo protestante Jürgen Moltmann en su libro "El camino de Jesucristo", nos permite leer en las estructuras profundas (subyacentes ) de este texto este complejo proceso al que aludimos en párrafos anteriores: " La Carta a los colosenses presenta una visión de paz universal basada en una "cristología cósmica". Por medio de Cristo, Dios "reconcilia consigo el universo, lo terrestre y lo celeste, para hacer la paz con su sangre derramada en la cruz" $(1,20)$, Cristo no murió únicamente en forma vicaria, la muerte de los hombres dolientes y la muerte del pecador para traer la paz al mundo humano; murió también la "muerte de todo ser vivo" para reconciliarlo todo en el cielo y en la tierra, es decir, incluidos los ángeles y los animales, y traer la paz a toda la creación. En virtud de su resurrección pasó a ser el "primero en nacer de la muerte" y se manifestó como el "nacido antes que toda criatura" $(1,15.18)$.

Lo que dice el antiguo testamento sobre la sabiduría de Dios como vida de la creación lo aplica la Carta a los colosenses al Cristo cósmico. Reconciliados por su muerte y acogidos en su nuevo nacimiento, todas las criaturas se benefician de la paz de la nueva comunidad universal. En Cristo toca a su fin el autoaislamiento de las distintas criaturas. En él toca a su fin la violencia. En él toca a su fin la injusticia. En él toca a su fin el poder de la muerte. En lugar de la destrucción mutua aparece una comunidad de paz en la que todos los seres conviven, se asisten y se implican, y conservan la vida en intercambio recíproco de energías. Cristo es "la cabeza" de esta nueva comunidad universal porque en él "habita realmente toda la plenitud de la divinidad" (Col 2, 9), y esta shekina fluye a través de él a la nueva comunidad universal, lo cual permite hablar con la teología ortodoxa de una "deificación del cosmos".

\section{La transformación/superación de sistema guerra/paz vía cristianismo}

Desde la más sencilla humildad franciscana, lejos de cualquier soberbia y vanidad, el aporte del Cristianismo a la paz es una vía posible -la única dirán los fieles- capaz de superar la escisión humana que ha generado el sistema con su movimiento perpetuo de guerra/paz. Recordemos lo que a propósito nos dice acertadamente nuestro ilustre colega, el Rev. José Miguel Torres Pérez(q.e.p.d): 


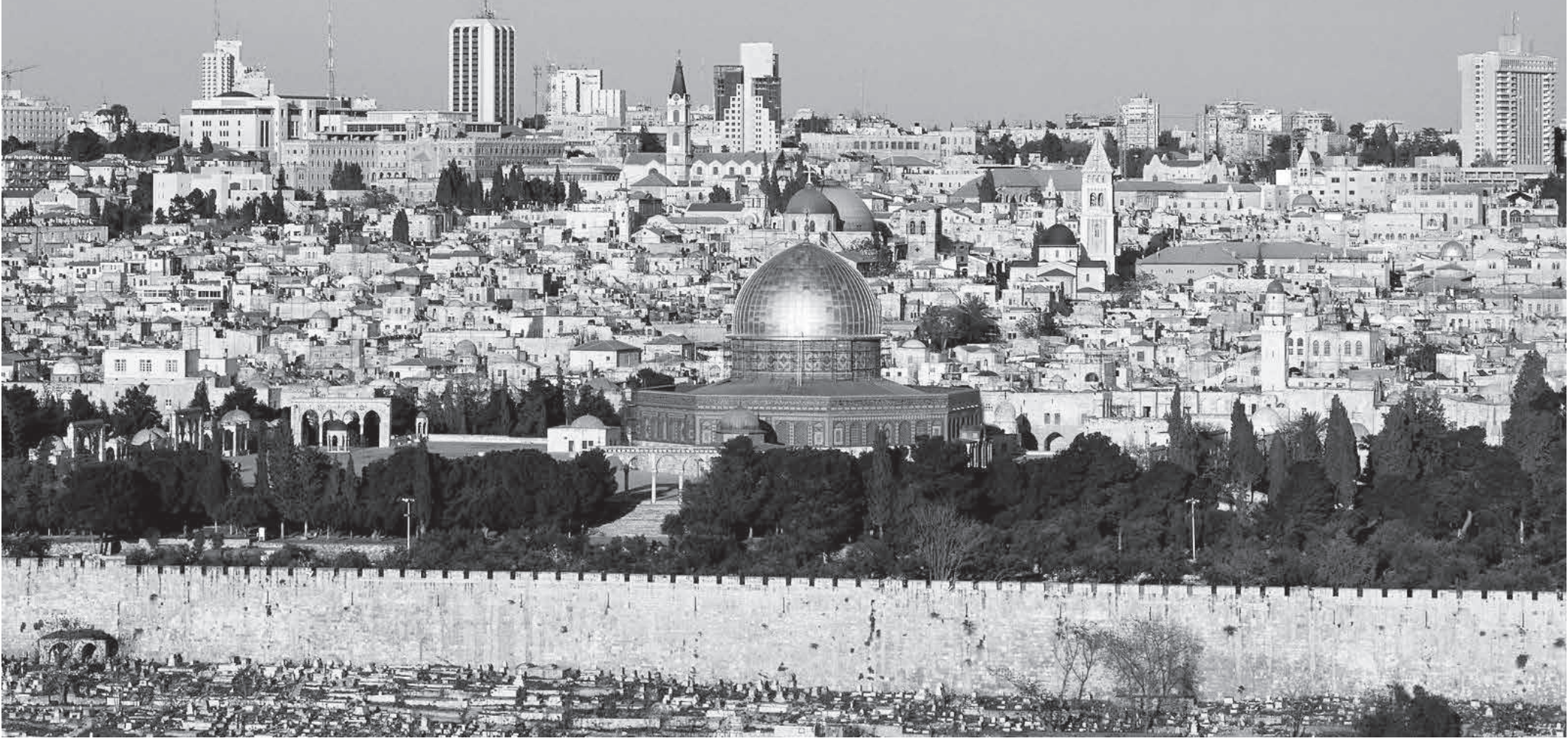

“La Biblia revela a Dios, como el Dios de Paz, a Jesús como el Príncipe de la Paz y al Espíritu Santo como el origen del amor, el gozo y la paz. Destaca el origen de Dios. Y llama a sus hijos a andar gobernados por su paz, anunciando la prosperidad y la armonía entre los hombres (según aparece el significado de Shallom en la bendición entre los judíos). La paz va más allá de un orden garantizado por una estructura de leyes o un estado obligado por la fuerza compulsiva de una autoridad militarizada. Procurarla, trabajar por ella, interceder ante Dios para que la conceda, participar esperando en aquel que tiene en sus manos las definiciones del mañana, es que nos ocupa, por eso estamos aquí, para construir la paz hemos sido convocados a la luz del Espíritu y la Palabra. Hay que rogar por ella a nuestro Dios Amén." (Torres 2003, p. $x x x)$

En relación a esta dimensión humana, divina, cósmica y cristocéntrica de la paz, no puedo dejar de apuntar que también en la Iglesia Católica se abunda en fundamentos sólidos y trascendentales. Baste citar para apuntalar nuestra afirmación este documente reciente de uno de los Papas titulado: Mensaje de SS Benedicto XVI para la Celebración de la XLIII Jornada Mundial de la Paz, 1 de Enero de 2010:

\section{“SI QUIERES PROMOVER LA PAZ, PROTEGE LA CREACIÓN"}

"14. Si quieres promover la paz, protege la creación. La búsqueda de la paz por parte de todos los hombres de buena voluntad se verá facilitada sin duda por el reconocimiento común de la relación inseparable que existe entre Dios, los seres humanos y toda la creación. Los cristianos ofrecen su propia aportación, iluminados por la divina Revelación y siguiendo la Tradición de la Iglesia. Consideran el cosmos y sus maravillas a la luz de la obra creadora del Padre y de la redención de Cristo, que, con su muerte y resurrección, ha reconciliado con 
Dios «todos los seres: los del cielo y los de la tierra» $(\mathrm{Col} 1,20)$. Cristo, crucificado y resucitado, ha entregado a la humanidad su Espíritu santificador, que guía el camino de la historia, en espera del día en que, con la vuelta gloriosa del Señor, serán inaugurados «un cielo nuevo y una tierra nueva» (2 P 3,13), en los que habitarán por siempre la justicia y la paz. Por tanto, proteger el entorno natural para construir un mundo de paz es un deber de cada persona. He aquí un desafío urgente que se ha de afrontar de modo unánime con un renovado empeño; he aquí una oportunidad providencial para legar a las nuevas generaciones la perspectiva de un futuro mejor para todos. Que los responsables de las naciones sean conscientes de ello, así como los que, en todos los ámbitos, se interesan por el destino de la humanidad: la salvaguardia de la creación y la consecución de la paz son realidades íntimamente relacionadas entre sí. Por eso, invito a todos los creyentes a elevar una ferviente oración a Dios, Creador todopoderoso y Padre de misericordia, para que en el corazón de cada hombre y de cada mujer resuene, se acoja y se viva el apremiante llamamiento: $\mathrm{Si}$ quieres promover la paz, protege la creación.

\section{BIBLIOGRAFÍA}

Moltmann, Jürgen: El camino de Jesucristo, Ed. Sígueme, Salamanca, 2000.

Muñoz, Francisco A. y López Martínez, Mario: Historia de la paz, Universidad de Granada, Granada, 2000.

Mensaje de SS Benedicto XVI para la Celebración de la XLIII Jornada Mundial de la Paz, 1 de Enero 2010.

Torres P., José Miguel: Bases y Fundamentos BíblicoTeológicos de una Cultura de Paz, Ed. Nos-Otros, IMLK- UPOLI, Managua, 2003.

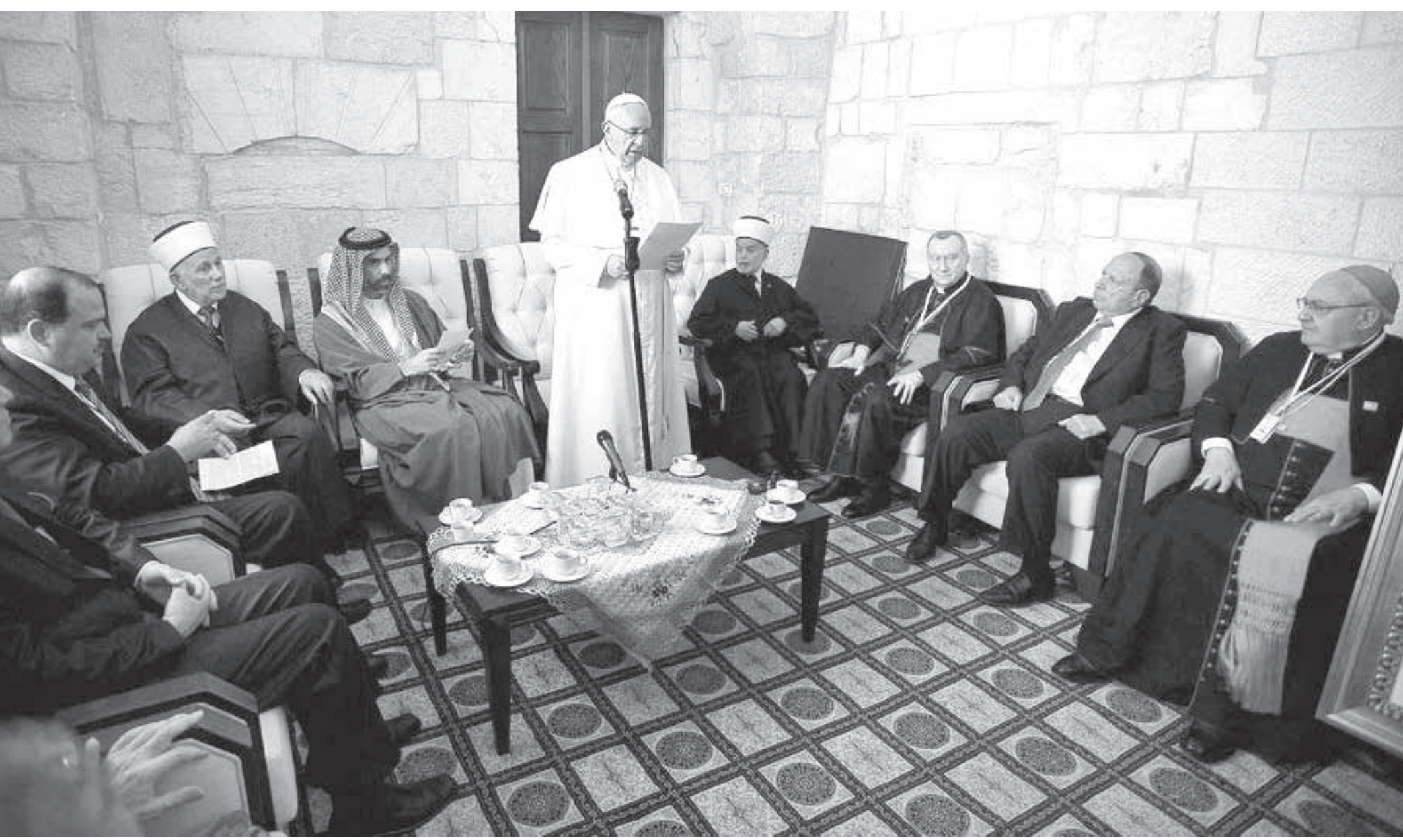

Fuente L’Ossevatore Romano 